\title{
Theoretical Study of Scanning Probe Microscope Images of $\mathrm{VTe}_{2}$
}

\author{
Sung Soo Park, ${ }^{+}$Jeevoung Lee, Wang Ro Lee, ${ }^{\ddagger}$ and Kee Hag Lee ${ }^{\circ}$ \\ Department of Chemistry, BK 21 Project, and Research Institute of Basic Sciences. Wonkitang Chiversitv. Iksan, \\ Jeonbuk 570-749. Korea. ${ }^{*}$ E-mail: khleeiâwonkwang.achr \\ -CAE Group. Central R \& D Institute. Samsung Electro-l lechancs Co. Lid., Sinwon 443-803. Korea \\ ${ }^{\ddagger}$ Department of Industrial Chemistry, Iksan National College, Iksan, Jeonbuk $570-752$. Korea \\ Received October 31.2006
}

\begin{abstract}
Ab initio periodic Hartree-Fock calculations with the full potential and ninimum basis set are applied to interpretation of scanning tunneling microscope (STM) and atomic force nicroscope (AFM) inages on IT. $\mathrm{VTe}_{\text {. }}$. Our results show that the simulated STM image shows asymmetry while the simulated AFM inage shows the circular electron densities at the bright spots without asymmetry of electron density to agree with the experimental AFM image. The bright spots of both the STM and AFM images of VTe, are associated with the surface Te atoms, while the patterns of bright spots of STM and AFM images are different.
\end{abstract}

Key Words : Scanning tunneling microscope image. Atomic force microscope image. IT-VTe2. Simulated STM and AFM images. Ab initio method

\section{Introduction}

Recently Scauning Probe Microscopes (SPMs) techniques including Scanning Tumeling Microscope (STM) and Atonic Force Microscope (AFM) have been applied to examine the surfaces of various solid materials. ${ }^{1}$ Layered materials. such as transition metal chalcogenides and graphite. are especially attractive as STM substrates for atomic scale imaging.

In the past decade. the experiments and theoretical interpretations in STM and AFM inage of transition metal chalcogenides have developed rapidly because these compounds are found to possess a rich structural chemistry and a wide variety of unusual physical properties. ${ }^{2 \cdot 4}$ The application of layered materials includes superconductivity and metallic conductivity. The intercalation properties of transition metal chalcogenides make it possible to extend the present knowledge about intercalation materials such as battery. ${ }^{6}$

The CdI: type of IT-MX $(\mathrm{M}=$ transition metal. $\mathrm{X}=$ chalcogen) have layers of composition $\mathrm{MX}_{2}$, which are constructed from $\mathrm{MX}_{6}$ octahedra by sharing their edges. ${ }^{7}$ In each $\mathrm{MX}_{2}$ layer. a slab of metal atoms is sandwiched between two slabs of chalcogen atonts. and the metal atoms of an undistorted $\mathrm{MX}_{2}$ layer form a hexagonal lattice. The $\mathrm{CdI}_{2}$ type IT-MX: phases with different $\mathrm{d}$ electron counts from $\mathrm{d}^{\mathrm{l}}$ to $\mathrm{d}^{3}$ exhibit various $\mathrm{M}-\mathrm{M}$ clustering patterns. ${ }^{7 \cdot 12}$ These phenomena often referred to as charge density waves (CDW). ${ }^{+12}$ Therefore. metal chalcogenides are attractive for AFM and STM study because individual layers are held by weak van der Waal's (VDW) interactions. The experimental images and theoretical interpretation of total electron density and partial electron density based on Extended Hückel Tight Binding (EHTB) method of STM and AFM are reported for IT-TaX2 $(X=S \text {. Se })^{+}$

The several crystal structure of $V \mathrm{Ve}_{2}$ is reported by distortion pattern at various temperature ${ }^{6.13}$. $\mathrm{VTe}_{2}$ has a $\mathrm{CdI}_{2}$ type structure above the transition temperature of $482 \mathrm{~K}$, while the structure is monoclinic with space group $C_{2 \text { in }}$ below $482 \mathrm{~K}$. The single crystal structures for $\mathrm{MTe}_{2}(\mathrm{M}=$ Ta. $\mathrm{Nb} . \mathrm{V}$ ) are observed by AFM experiments. ${ }^{1+}$ The observed AFM images for $\mathrm{MTe}_{2}(\mathrm{M}=\mathrm{Ta}, \mathrm{V})$ were analyzed by calculating the total electron density plots on the basis of EHTB method. ${ }^{15}$ The electronic structures for the $\mathrm{CdI}_{2}-t y p e$ layered transition metal chalcogenides $\left[\operatorname{TiX}_{2}(X=\mathrm{S}\right.$. Se. Te), $\left.\mathrm{MTe}_{2}(\mathrm{M}=\mathrm{V}, \mathrm{Nb}, \mathrm{Ta})\right]$ are calculated by Extended Hückel Tight Binding (EHTB) method. ${ }^{16}$

In the present work. we analyze the AFM and STM images of the layered monoclinic IT-VTe 2 . by using $a b$ initio Periodic Hartree-Fock calculations in the CRYSTAL95 program. ${ }^{17}$

\section{Calculations}

We used $a b$ initio periodic Hartree-Fock (PHF) calculations with the minimum basis set in the CRYSTAL95 program to study a monoclinic $\left(\mathrm{C}_{2: \mathrm{m}}\right) \mathrm{VTe}_{2}$ slab model with two layers. The periodic Hartree-Fock (PHF) method systematically expands the band structure, whereas the density functional theory (DFT) systematically underestimates band gaps and band widths. The computer code CRYSTAL95 is nowadays one of the standard tools for theoretical investigations in the field of material science to explain chemical and physical properties.

Applying a small bias voltage $\mathrm{V}$ between the tip and the sample yields a tunneling current. The ideal STM would have the greatest possible resolution and would measure the intrinsic properties of unperturbed surfaces rather than the properties of the joint surface-tip interface. A tip whose potential and wave functions were arbitrarily chosen would best achieve this ideal condition. In that case. Tersoff and Hamann $^{18}$ have considered the limit of the point probe. In the small bias voltage limit, the tumneling conductance, $\sigma$ is 
where

$$
\sigma \propto \rho_{S T M}(r, V)
$$

and

$$
\rho_{S T M}(r, V)=\int_{E_{F-Q F}}^{E_{F}} d E \sigma(r, E)
$$

and

$$
\rho(r, E) \equiv \Sigma_{v}\left|\psi_{v}(r)\right|^{2} \delta\left(E_{v^{\prime}}-E\right)
$$

where $r$ is in the position of the center of curvature of the tip s state, $E_{F}$ is the Fermi energy, $\rho(r, E)$ is the surface local density of states (LDOS) of the bare surface at the center of the tip $r=(x, y, z)$ and $\psi_{v}(r)$ are the electron eigenstates of the unperturbed surface with corresponding energy $E_{1}$. For a periodic system. the sum of the $v$ state is a sum of the bands and an integral of the $k$ spacing spanning the irreducible part of the Brillouin zone. In CRYSTAL95. a regular mesh of interpolating points is used for performing the numerical integration in the reciprocal space. The details of the $k$ integration scheme can be found in ref 19. The implied assumptions are that the description of the relevant tip states is given by a locally spherical potential well with a constant density of states where it approaches nearest to the surface with a constant density of states. and that the tunneling matrix element is independent of the lateral tip position for a constant tip-to-position and also is independent of the bias voltage $V$ in the narrow energy region $\left[E_{F} \pm \mathrm{eV} . E_{F}\right)$.

The calculation of STM charge density. $\rho_{S T R}(r, V)$ is performed by sampling the k-space region near the Fermi surface in the irreducible part of surface Brillouin zone (BZ). Not all the $k$ points contribute to $\rho_{\operatorname{STM}}(r, V)$, because the portion of the band structure sampled by the STM is very small for typical tumeling voltage below $\sim 1 \mathrm{~V}$. In contact mode AFM measurements. all the electrons of the surface atoms are involved in the repulsive interactions with the tip. so that the AFM image is described by the total electron density plot $\rho_{A F, 1}(r)$ of the surface

Here. we calculate the STM charge density within bias voltage $1.0 \mathrm{~V}$ at tip-to-surface separation of $1.0 \mathrm{~A}$. And we calculate the AFM charge density at tip-to-surface separation of $1.0 \mathrm{~A}$. The crystal structure of $\mathrm{VTe}_{2}$ is chosen from ref. 6 .

\section{Results and Discussion}

The STM and AFM inages are simulated. Here we calculate STM charge densities $\rho_{S T M}(r . V)$ defined in Eq. (2) and AFM charge densities $\rho_{A F M}(Y)$. In other words, the STM charge density is described by the partial electron density, while the AFM charge density is described by the total electron density. Due to the phase transition of hexagonal IT$\mathrm{VTe}_{2}$ (Figure I) to monoclinic IT-VTe 2 (Figures 2 and 3) by temperature. each monoclinic $1 \mathrm{~T}-\mathrm{VTe}$ (thereafter referred to $\mathrm{VTe}_{2}$ ) layer has two non-equivalent $\mathrm{V}$ atoms and three nonequivalent $\mathrm{Te}$ atoms ${ }^{8}$ which form the ribbon chain. Figure 2 shows a top projection view in the $a b$ plane of a single VTe. layer. Figure 3 shows a side projection view in the $a c$ plane

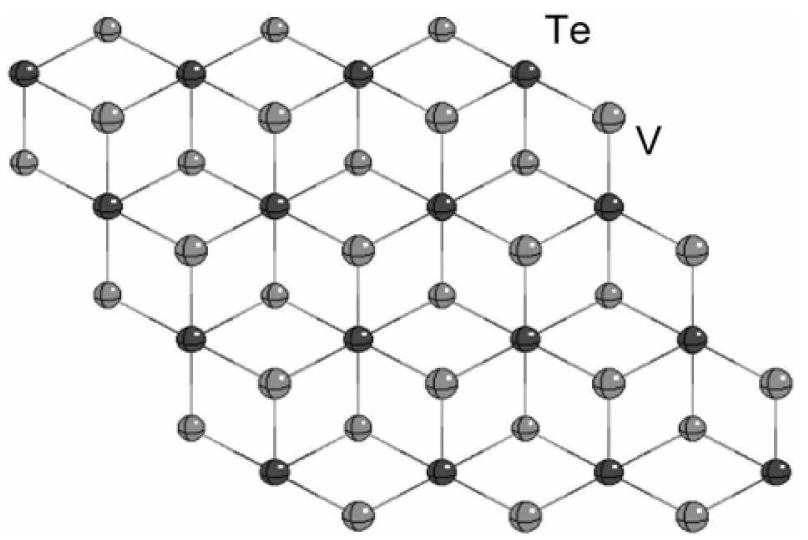

Figure 1. Schematic projection view of undistorted lT-MX 2 (ideal C.II type structure).

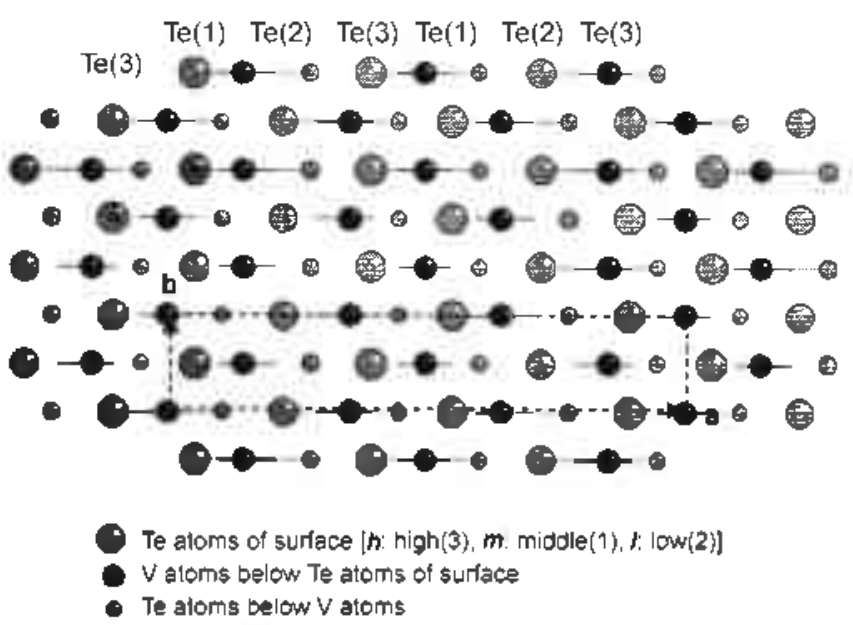

Figure 2. $(001)$ surface of monoclinic VTe.

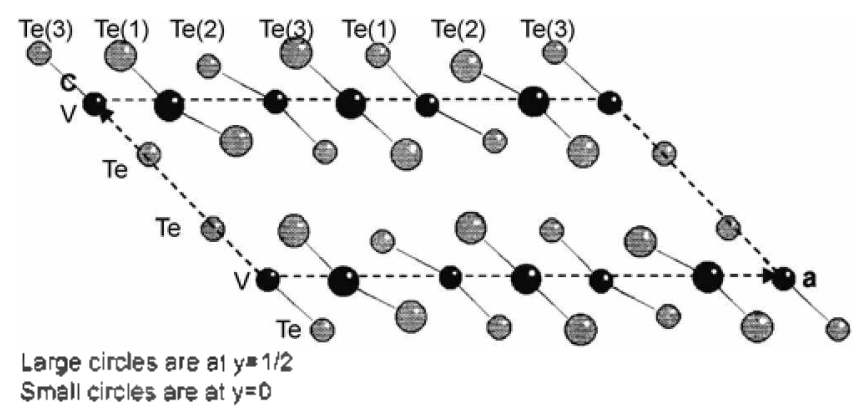

Figure 3. Projection of the structure on the $(010)$ plane of monoclinic $\mathrm{VTe}_{2}$.

of two adjacent $\mathrm{VTe}_{2}$ layer along the ribbon chains which nun parallel to the $b$-direction.

As shown in Table 1. the $\mathrm{Te}(3)$ atoms protrude the highest on the surface and they lie higher than the $\mathrm{Te}(1)$ and $\mathrm{Te}(2)$ atoms by $0.488 \AA$ and $0.086 \AA$. respectively. Figure 4 shows the $\rho_{A F A}(r)$ plot that has the electron density distribution on the $a b$-plane surface. In each unit cell of the $\rho_{A F M}(r)$, the electron density of the Te atom decreases in the order. Te(3) $>\mathrm{Te}(2)>\mathrm{Te}(1)$. This is consistent with the finding that the distances of the surface Te atoms to the tip are increasing in the order: $\operatorname{Te}(3)<\operatorname{Te}(2)<\operatorname{Te}(1)$. Consequently the $\rho_{\text {. }}$ : $1 /(r)$ 
Table 1. Atomic distances $(\hat{A})$ of $V T_{2}$ with monoclinic structure $(2 / m$

\begin{tabular}{|c|c|c|c|}
\hline & Exp. & calc. (I) & calc. (2) \\
\hline$V(1)-V(2)$ & $3.316(8)$ & 3.316 & 3.318 \\
\hline$V(2)-V(2)$ & $4.32(1)$ & 4.316 & 4.318 \\
\hline \multicolumn{4}{|c|}{ M-X distances in the centric octahedron } \\
\hline$V(1)-T e(1)$ & $2.718(2)$ & 2.718 & 2.718 \\
\hline$V(1)-T e(3)$ & $2.743(5)$ & 2.743 & 2.740 \\
\hline \multicolumn{4}{|c|}{ M-X distances in the acentric octahedron } \\
\hline$V(2)-T e(1)$ & $2.58(1)$ & 2.583 & 2.58 \\
\hline$V(2)-T e(2)$ & $2.733(5)$ & 2.732 & 2.734 \\
\hline$V(2)-T e(2)$ & $2.79(1)$ & 2.790 & 2.789 \\
\hline$V(2)-T e(3)$ & $2.661(4)$ & 2.660 & 2.661 \\
\hline \multicolumn{4}{|c|}{$\mathrm{X}$-X distances parallel to layers } \\
\hline $\operatorname{Te}(1)-\operatorname{Te}(2)$ & $3.744(5)$ & 3.742 & 3.744 \\
\hline $\operatorname{Te}(2)-\operatorname{Te}(3)$ & $3.737(5)$ & 3.738 & 3.741 \\
\hline $\operatorname{Te}(1)-\operatorname{Tc}(3)$ & $3.492(5)$ & 3.492 & 3.494 \\
\hline \multicolumn{4}{|c|}{$\mathrm{X}$-X distances between to layers } \\
\hline $\operatorname{Te}(1)-\operatorname{Te}(1)$ & $3.59(3)$ & 3.590 & 3.588 \\
\hline$T e(1)-T c(2)$ & $3.907(6)$ & 3.907 & 3.901 \\
\hline $\mathrm{Te}(2)-\mathrm{Tc}(3)$ & $3.778(3)$ & 3.778 & 3.776 \\
\hline $\operatorname{Te}(3)-\operatorname{Te}(3)$ & $3.595(6)$ & 3.595 & 3.591 \\
\hline \multicolumn{4}{|c|}{$\mathrm{X}$-X distances vertical to layers } \\
\hline $\operatorname{Te}(1)-\operatorname{Te}(3)$ & & 4.077 & 4.075 \\
\hline $\operatorname{Te}(1)-\mathrm{Te}(3)$ & & 4.198 & 4.193 \\
\hline $\operatorname{Te}(2)-\mathrm{Te}(2)$ & & 3.447 & 3.443 \\
\hline $\mathrm{Te}(2)-\mathrm{Tc}(3)$ & & 3.947 & 3.946 \\
\hline
\end{tabular}

In calc. (1) unit cell paraneers: a - 18.984, b-3.5947. c-9.069. $\beta-$ $134.62^{\circ} \omega$ of Sold State (hem. 1984. 53. 415$)^{13}$ In calc. (2). unit cell parameters: $\mathrm{a}=18.992 . \mathrm{b}=3.5982 . \mathrm{c}=9.0585, \beta=134.64^{\circ} \mathrm{H}$. of Waterial (hem. 1993, 3, 127I)."

plot of Figure 4 predicts that the bright spots of the ArM images are caused by the surface Te atoms. This plot is presented by the same pattern consisting of the previous experimental image and theoretical calculation." "Figure 5 shows for the atomic scale STM image of VTe $\mathrm{V}_{2}$ which is characterized by rows of bright spots.

It is very difficult that we observe the STM image of $\mathrm{VIe}_{2}$, but we can show rearrangement of high electron density in experimental result. The $\rho_{7,1}(r, V)$ plot of the ab-plane (Figure 5) is considerably different from the $\rho_{+1}(r)$ plot of Figure 4. The partial electron density on $\mathrm{Te}(1)$ and $\mathrm{Te}(3)$ atom is not spherical but represents in-plane 5 p-orbital density. The crystal structure obtained from $\mathrm{X}$-ray diffraction experiment which interlayer Te-Te distance of $a b$ plane is about $3.6 \AA$ whereas the distance between high electron density sites is about $2 \AA$ to b-direction in the preliminary STM image experiment. ${ }^{20}$ Our calculation reveals the $\rho_{m} \mathrm{M}(r$, $n$ plot which has a unit cell of rectangle of about $3.6 \dot{\AA}$ to $a$ direction and about $2.0 \AA$ to $b$-direction. Thus, our calculation would be useful to explain qualitatively the preliminary image of SIM experiment. But the vacuum SIM image for $\mathrm{VTe}_{2}$ is not obtained yet, which is necessary to compare quantitatively with our SIM image obtained by the simulation.

In conclusion, our calculations suggest that the bright

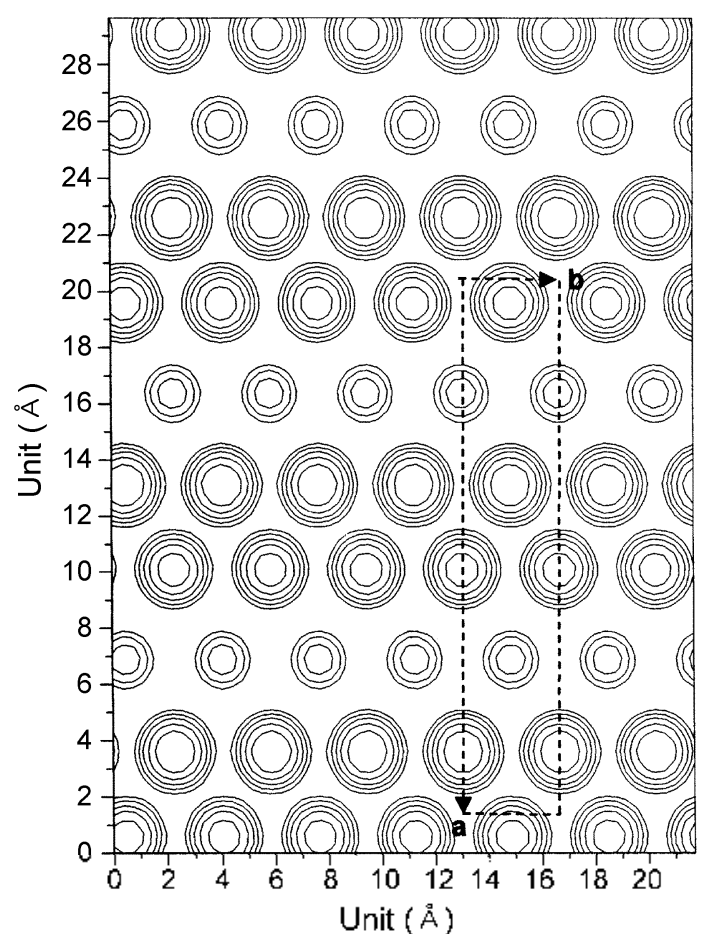

Figure 4. Contour plot of the

$\Lambda \mathrm{M}$ charge density in plane parallel to and $\mathrm{I} \Lambda$ above the topmost surface layer of monoclinic VTe. Consecutive contours from minimum isolines (0.01) are separated up to the maximum values $(0.08)$.

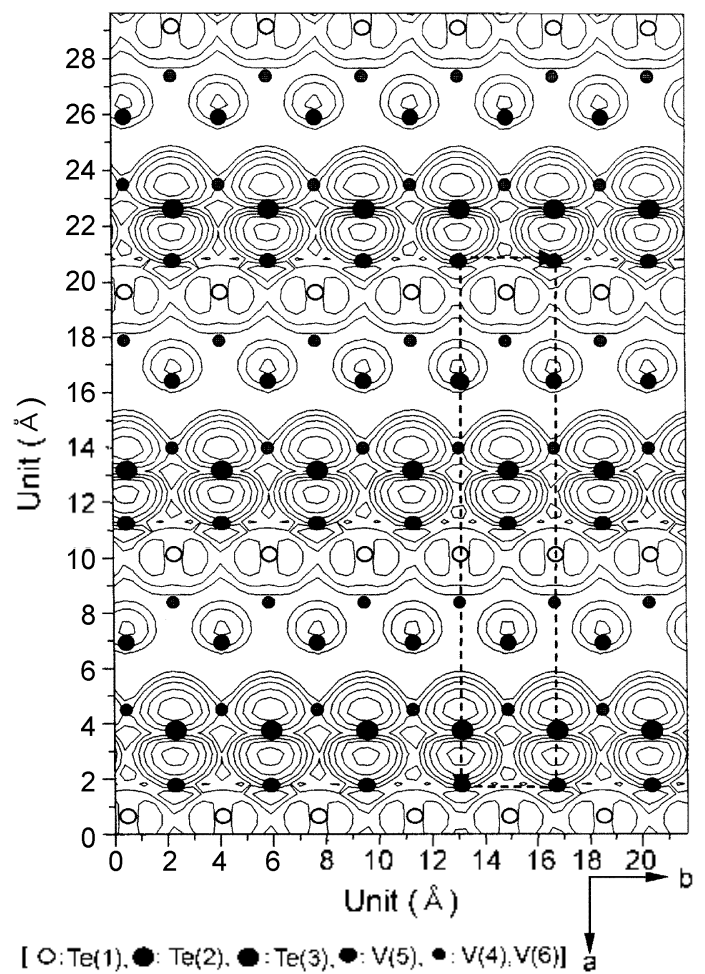

Figure 5 . Contour plots of the SIM charge density $p_{s, n}$ in plane parallel to and I $\mathbb{A}$ above the topmost surface layer of monoclinic $\mathrm{VTe}_{2}$. Surlace result for gly $_{7}$ with the tip-1o-surface separation $1 \AA$ is given lor bias voltages $1.0 \mathrm{~V}$. The units are $10^{-6}$ electrons/an ${ }^{3}$, and consecutive contours from minimum isolines are separated with 50 steps up to $1.0 \times 10^{-4}$ maximum isolines. 
spots of both the STM and AFM intages of VTe: are associated with the surface Te atoms. The STM image shows a pattern of three $\mathrm{Te}$ atoms of $\mathrm{Te}(1) \mathrm{Te}(2)$, and $\mathrm{Te}(3)$ in which $\mathrm{Te}(3)$ has the highest electron density. $\mathrm{Te}(2)$ and $\mathrm{Te}(3)$ sites show the electron density morphology of asymmetrical dumbbell. while $\mathrm{Te}(\mathrm{l})$ sites show the electron density morphology of very weak asymmetrical dumbbell. The AFM image possesses a circle-type pattern for three kinds of $\mathrm{Te}$ atoms.

Acknowledgement. This work was supported by Wonkwang University in 2004. We thank Prof. H. Kang at Seoul National University for showing us his unpublished STM image of $\mathrm{VTe}_{2}$ and stimulating discussions.

\section{References}

1. Wiesendander. R. Scanning Probe Microscopy and Spectroscopv: Cambridge University: Cambridge. 1994. Wiesendander. R: Anselmetti. D. Suface Properties and Lanend Strachmes: Kluwer: The netherlands, 1992. Magonov S. N.: Whangbo, M.-H. Surface Analysis with STM and AFM: VCH: Weinheim: Germany: 1996. Halm, I. R. Bull. Korean (Them. Soc. 2005, 26(7), 1071.

2. (a) Parkinson. B. A.: Ren. J.: Whangbo. M.-H. J. Am. Chem, Soc. 1991. 113. 7833. (b) Mar. A .: Jobic, S.: Ibers. J. A. J. Am. Chem. Soc. 1992. HH. 8963. (c) Ren. J.: Whangbo. M.-H.: Bengel. H.: Magonov. S. N. J. Phns. Chem. 1993. 97, 4764. (d) Ren. J.: Whangbo, M.H.: Bengel. H.: Cantow. H. I.: Magonov. S. N. Chem. Hater. 1993. 7, 1018. (e) Ren, J.: Whangbo. M. H: Zonnchen, P.: Rotter, H. Cantow: H. J: Thiele. G. Magonot. S. N. J. Am. Chem. Soc. 1993. 15. 2495

3. Hulliger. F. Simctiral Chemistry of Laver-tppe Phases. Lèvy. F. Ed.: Reidel: Dordrecht-Holland. 1976.

4. (a) Whangbo. M. H.: Canadell, E. J. Am. Chem. Soc. 1992. 114. 9587. (b) Brouwer, R: Jellinek: F. Phnsica B 1980. 99. 51

5. (a) Wilson. J. A.: DiSalbo. F. J: Mahajan. S. Ad, Phns. 1975 , 24.
117. (b) Sorgel, T: Jansen, M. Solid State Sci. 2004, 6. 1259.

6. Williams. P. M. Crnstallography and Cnstal Chemistiy of Materials with Lavered Structures: Lèv. F.. Ed.: Reidel: DordrechtHolland. 1976.

7. Snucture Phase Transitions in Lavered Transition Metal Compounds: Motzuki. K.. Ed.; Reidel: Dordrecht-Holland. 1986.

8. Doni. E: Girlanda, R. Electronic Structure and Electronic Transition in Lavered Materials: Grasso. V. Ed: Reidel: DordrechtHolland. 1986.

9. Whangbo. M. H.: Ret1. J.: Canadell. E.: Louder. D.: Pakinson. B A.: Bengel. H: Magonov, S. N. J.Am. Chem. Soc. 1993, 115.3760.

10. Ishihara, Y; Nakada, I. Solid State Commun. 1982, 42.579

11. Guzmán. R.: Morales. T.: Tirado. T. L. J. Mater. Chem. 1993. 3. 1271 .

12. Bronsema. K. D.: Bus. G. W.: Wiegers. G. A. J. Solid State Chent. 1984. 53. 415

13. Kim. S. J.: Park, S. J.: Oh, H. J.; Jeon. I. C.: Song, S. Bull. Kor: Chem. Soc, 1994, 15. 1098.

14. Kim. S. J.: Park. S. J.: Jeon. I. C.: Kim. C.: Pyun, C.: Yee. K. A. d. Phis Chem. Solids 1997. 58.659.

15. Canadell. E.: Tobic. S.: Brec. R.: Rousel. T.: Whangbo. M. H. J. Solld State Chem. 1992.99. 189

16. Coleman. R. V: Giambattista. B.: Hansma, P. K.; Jolnnson, A.: MeNairy, W. W. Slough, C. G. Adv Plns. $1988,37,559$.

17. Dovesi. R.: Saunders, V. R.: Roetti. C.: Causa, M.: Harrison, N. M.: Orlando. R: Apra. E. CRYSTLL95 User's Hamat: Univ. of Torino: Torino. Italy: 1996.

18. (a) Tersoft. T.: Hamann. D. R. Phs. Rev Lett. 1983.50. 1998. (b) Tersoff. J.: Hamann, D. R. Plns. Rev B 1985, 31. 805 . (c) Lee. K. H.; Causá, M.: Park. S. S. J. Phys. Chem. $B$ 1998. 102, 6020. (d) Lee. K. H.: Causa. M; Park, S. S.; Lee. C.: Suh. Y.: Eun, H. M.; Kim. D. J. Molecular Stuc. (Theochem I 2000, 297,301.

19. (a) Pisani. C.: Dovesi. R.: Roetti. C. Hortree-Fock $a b$ intio Treament of Chutalline Stutents: Springer-Verlag: Heidelberg. 1988. (b) Pisani. C.: Apra. E.: Causa. M. J. Quontum Chem. 1990. 38. 395. (c) Pisani C: Apra. E.: Causa. M: Orlando, R. $d$. Ouanum Chem. 1990. 38. 419 .

20. Unpublished result of a STM image for VTe 2 from Prof. Heon Kang at Secul National University. 